\title{
Differential Mobility Spectrometry Coupled with Multiple Ion Monitoring in Regulated LC-MS/MS Bioanalysis of a Therapeutic Cyclic Peptide in Human Plasma
}

Yunlin Fu ${ }^{1}$, Yuan-Qing Xia ${ }^{2} *$, Jimmy Flarakos ${ }^{1}$, Francis L.S. Tse ${ }^{1}$, Jeffrey D. Miller ${ }^{2}$, Elliott B. Jones $^{2}$, Wenkui $\mathrm{Li}^{1}{ }^{2}$

${ }^{1}$ Early Bioanalytics and Technology, Drug Metabolism and Pharmacokinetics, Novartis Institutes for BioMedical Research, One Health Plaza, East Hanover, NJ 07936. ${ }^{2}$ Sciex, 500 Old Connecticut Path, Framingham, MA 01701

*Corresponding authors: ${ }^{1}$ Wenkui Li: wenkui.li@ novartis.com; Phone: 862-778-4255, Fax: 973781-7579; ${ }^{2}$ Yuan-Qing Xia: shaw.xia@ sciex.com; Phone: 609-423-9410, Fax: 609-423-9410

\section{Content}

- A short description of method validation for the LC-MRM determination of pasireotide in human plasma with a dynamic range of 0.1 to $50 \mathrm{ng} / \mathrm{mL}$ using a volume of $100 \mu \mathrm{L}$ human plasma

- Accuracy and precision of the QC samples in spiked human plasma, Table S-1

- Post-preparative stability in human plasma sample extracts with the autosampler set at $5^{\circ} \mathrm{C}$, Table S-2

- Freeze-thaw stability in human plasma samples stored at or below $-60^{\circ} \mathrm{C}$, Table S-3

- Stability in spiked human plasma samples stored at room temperature, Table S-4

- Stability in spiked human plasma samples stored at or below $-60^{\circ} \mathrm{C}$, Table S-5

- Carryover in human plasma blanks following injection of the ULOQ for pasireotide, Table S-6

- Carryover in human plasma blanks following injection of the ULOQ for $[\mathrm{M}+6]$ pasireotide, Table S-7

- Stability of pasireotide in stock solution, Table S-8

- Matrix effect for pasireotide, Table S-9

- Recovery for pasireotide, Table S-10

- A comparison of LC-DMS-MIM response for the pasireotide with and without chemical modifier isopropanol, Figure S-1 


\section{A short description of method validation for the LC-MRM determination of pasireotide in human plasma with a dynamic range of 0.1 to $50 \mathrm{ng} / \mathrm{mL}$ using a volume of $0.1 \mathrm{~mL}$ human plasma}

All calculations were done in Watson LIMS (Thermo Fisher Scientific, Philadelphia, PA, U.S.A) and/or Microsoft Excel 2003/2010 using validated spreadsheets.

Acceptance criteria. All bias and CV acceptance criteria were set at $\leq 15 \% \mathrm{CV}(\leq 20 \%$ at the LLOQ) and within $\pm 15 \%$ bias ( $\pm 20 \%$ at the LLOQ), except for the stock comparison and stock stability assessments, for which $\pm 10 \%$ difference limits were used.

Linearity. Each validation run contained two sets of calibration standards prepared in human plasma at the following concentration levels: 0.1, 0.2, 0.4, 0.8, 4, 20, 40 and $50 \mathrm{ng} / \mathrm{mL}$. The ratio of the measured peak area of the analyte over that of the internal standard was used for the calibration regression. Weighted linear regression was applied with $1 / \mathrm{x}^{2}$ as weighting factor.

Accuracy and precision. The accuracy and precision of the method were determined by three separate runs (validation core runs) analyzed on three different days. The QC samples were prepared in human plasma at five concentration levels: 0.1 (LLOQ), 0.3, 1.5, 15 and $37.5 \mathrm{ng} / \mathrm{mL}$.

Stability in the sample extract in the autosampler. Human plasma samples at concentrations of 0.3 (QC low) and $37.5 \mathrm{ng} / \mathrm{mL}$ (QC high) were extracted and analyzed in the first validation run as a part of the accuracy and precision assessment. Subsequently, the extracts were kept in the autosampler set at $5^{\circ} \mathrm{C}$ for 5 days, after which they were re-analyzed for analyte concentrations. The observed bias (\%) of within $\pm 15 \%$ of nominal values is considered acceptable.

Freeze-thaw stability. Human plasma samples at concentrations of 0.3 (QC low) and 37.5 $\mathrm{ng} / \mathrm{mL}$ (QC high) were stored at $\leq-60^{\circ} \mathrm{C}$ and subjected to three freeze $\left(\leq-60^{\circ} \mathrm{C}\right.$ )-thaw (room temperature) cycles. The samples were analyzed against freshly prepared calibration standards. The observed bias $(\%)$ of within $\pm 15 \%$ of nominal values is considered acceptable.

Bench-top stability of the analyte in human plasma. The bench-top stability of analyte in human plasma was evaluated at ambient temperature $\left(\sim 22^{\circ} \mathrm{C}\right)$ over 24 hours using QC samples at concentrations of 0.3 (QC low) and $37.5 \mathrm{ng} / \mathrm{mL}$ (QC high). The measured concentrations of analyte in these QC samples were compared to the nominal values. The observed bias (\%) of within $\pm 15 \%$ of nominal values is considered acceptable.

Storage stability. Human plasma samples at concentrations of 0.3 (QC low) and $37.5 \mathrm{ng} / \mathrm{mL}$ (QC high) were stored at $\leq-60^{\circ} \mathrm{C}$ for 142 days. The samples were analyzed against calibration standards freshly prepared from the new stock solution. The observed bias $(\%)$ of within $\pm 15 \%$ of nominal values is considered acceptable.

Carry-over. For each of the validation runs, two blank samples were placed immediately after the upper limit of quantification (ULOQ) standard sample to evaluate the potential 
carryover. A LC-MS/MS response from the first blank sample injection of $\leq 20 \%$ of that of the LLOQ from the same run is considered acceptable.

Stock comparison and stock stability assessment. Two $1.00 \mathrm{mg} / \mathrm{mL}$ stock solutions of the analyte was prepared and compared against each other. 268 days later, two new stock solutions were prepared and compared against each other and also against the old stocks. A difference of $\leq 10 \%$ in LC-MS/MS response between the new and old stocks is considered acceptable.

Assessment of matrix effect and recovery. The matrix effect was estimated by spiking the analyte neat solutions into blank plasma sample extracts prepared from six individual lots with analyte at 0.3 (low), 15 (mid) and 37.5 (high) $\mathrm{ng} / \mathrm{mL}(\mathrm{n}=3)$ concentration levels. The matrix factors were calculated by comparing the peak areas of analyte in these post-spiked sample extracts with those in the corresponding neat solutions. The recovery was estimated by comparing the mean peak areas of the analyte in the extracted QC samples at 0.3 (low), 15 (mid) and 37.5 (high) $\mathrm{ng} / \mathrm{mL}(\mathrm{n}=3)$ with those obtained from extracted blank plasma samples post-spiked with the corresponding neat solutions. 
Table S-1 Accuracy and precision of the QC samples in spiked human plasma

\begin{tabular}{|c|c|c|c|c|c|c|c|c|c|c|c|}
\hline \multirow[t]{3}{*}{ Run Date } & \multirow{3}{*}{$\begin{array}{l}\text { Run } \\
\#\end{array}$} & \multicolumn{10}{|c|}{ Pasireotide nominal concentration $(\mathrm{ng} / \mathrm{mL})$} \\
\hline & & $\begin{array}{l}\text { Low } \\
(0.100)\end{array}$ & $\begin{array}{l}\% \\
\text { Bias }\end{array}$ & $\begin{array}{l}\text { Mid } \\
(0.300)\end{array}$ & $\begin{array}{l}\% \\
\text { Bias }\end{array}$ & $\begin{array}{l}\text { Mid } \\
(1.50)\end{array}$ & $\begin{array}{l}\% \\
\text { Bias }\end{array}$ & $\begin{array}{l}\text { Mid } \\
(15.0)\end{array}$ & $\begin{array}{l}\% \\
\text { Bias }\end{array}$ & $\begin{array}{l}\text { High } \\
(37.5)\end{array}$ & $\begin{array}{l}\% \\
\text { Bias }\end{array}$ \\
\hline & & \multicolumn{10}{|c|}{ Calculated concentrations ( $\mathrm{ng} / \mathrm{mL}$ ) and individual bias (\%) } \\
\hline & 1 & 0.101 & 1.0 & 0.316 & 5.3 & 1.60 & 6.7 & 15.8 & 5.3 & 38.7 & 3.2 \\
\hline & & 0.111 & 11.0 & 0.325 & 8.3 & 1.59 & 6.0 & 15.2 & 1.3 & 37.0 & -1.3 \\
\hline & & 0.0991 & -0.9 & 0.313 & 4.3 & 1.60 & 6.7 & 16.2 & 8.0 & 38.1 & 1.6 \\
\hline & & 0.105 & 5.0 & 0.314 & 4.7 & 1.64 & 9.3 & 15.5 & 3.3 & 38.1 & 1.6 \\
\hline & & 0.106 & 6.0 & 0.320 & 6.7 & 1.54 & 2.7 & 16.0 & 6.7 & 38.3 & 2.1 \\
\hline & & 0.0900 & -10.0 & 0.311 & 3.7 & 1.53 & 2.0 & 15.3 & 2.0 & 38.7 & 3.2 \\
\hline Intrarun Mean & & 0.102 & & 0.317 & & 1.58 & & 15.7 & & 38.2 & \\
\hline Intrarun SD & & 0.00720 & & 0.00517 & & 0.0413 & & 0.398 & & 0.625 & \\
\hline Intrarun \% CV & & 7.1 & & 1.6 & & 2.6 & & 2.5 & & 1.6 & \\
\hline Intrarun \% Bias & & 2.0 & & 5.7 & & 5.3 & & 4.7 & & 1.9 & \\
\hline \multirow[t]{7}{*}{$\mathrm{n}$} & & 6 & & 6 & & 6 & & 6 & & 6 & \\
\hline & 2 & 0.0977 & -2.3 & 0.282 & -6.0 & 1.61 & 7.3 & 15.3 & 2.0 & 38.8 & 3.5 \\
\hline & & 0.0958 & -4.2 & 0.294 & -2.0 & 1.54 & 2.7 & 15.1 & 0.7 & 36.6 & -2.4 \\
\hline & & 0.108 & 8.0 & 0.305 & 1.7 & 1.60 & 6.7 & 15.5 & 3.3 & 38.5 & 2.7 \\
\hline & & 0.0986 & -1.4 & 0.322 & 7.3 & 1.49 & -0.7 & 15.6 & 4.0 & 37.7 & 0.5 \\
\hline & & 0.0999 & -0.1 & 0.307 & 2.3 & 1.50 & 0.0 & 15.2 & 1.3 & 37.0 & -1.3 \\
\hline & & 0.0936 & -6.4 & 0.294 & -2.0 & 1.52 & 1.3 & 14.9 & -0.7 & 37.5 & 0.0 \\
\hline Intrarun Mean & & 0.0989 & & 0.301 & & 1.54 & & 15.3 & & 37.7 & \\
\hline Intrarun SD & & 0.00496 & & 0.0138 & & 0.0509 & & 0.258 & & 0.847 & \\
\hline Intrarun \% CV & & 5.0 & & 4.6 & & 3.3 & & 1.7 & & 2.2 & \\
\hline Intrarun \% Bias & & -1.1 & & 0.3 & & 2.7 & & 2.0 & & 0.5 & \\
\hline \multirow[t]{7}{*}{$\mathrm{n}$} & & 6 & & 6 & & 6 & & 6 & & 6 & \\
\hline & 3 & 0.0927 & -7.3 & 0.303 & 1.0 & 1.44 & -4.0 & 14.5 & -3.3 & 37.4 & -0.3 \\
\hline & & 0.0914 & -8.6 & 0.305 & 1.7 & 1.49 & -0.7 & 14.9 & -0.7 & 36.1 & -3.7 \\
\hline & & 0.0898 & -10.2 & 0.295 & -1.7 & 1.45 & -3.3 & 15.1 & 0.7 & 37.4 & -0.3 \\
\hline & & 0.0914 & -8.6 & 0.294 & -2.0 & 1.48 & -1.3 & 15.7 & 4.7 & 37.0 & -1.3 \\
\hline & & 0.0913 & -8.7 & 0.276 & -8.0 & 1.49 & -0.7 & 14.7 & -2.0 & 37.2 & -0.8 \\
\hline & & 0.0981 & -1.9 & 0.288 & -4.0 & 1.54 & 2.7 & 15.1 & 0.7 & 35.5 & -5.3 \\
\hline Intrarun Mean & & 0.0925 & & 0.294 & & 1.48 & & 15.0 & & 36.8 & \\
\hline Intrarun SD & & 0.00292 & & 0.0106 & & 0.0354 & & 0.415 & & 0.787 & \\
\hline Intrarun \% CV & & 3.2 & & 3.6 & & 2.4 & & 2.8 & & 2.1 & \\
\hline Intrarun \% Bias & & -7.5 & & -2.0 & & -1.3 & & 0.0 & & -1.9 & \\
\hline $\mathrm{n}$ & & 6 & & 6 & & 6 & & 6 & & 6 & \\
\hline $\begin{array}{l}\text { Overall } \\
\text { mean }(\mathrm{ng} / \mathrm{mL})\end{array}$ & & 0.0978 & & 0.304 & & 1.54 & & 15.3 & & 37.5 & \\
\hline Inter-run SD & & 0.00647 & & 0.0140 & & 0.0590 & & 0.443 & & 0.926 & \\
\hline Inter-run \% CV & & 6.6 & & 4.6 & & 3.8 & & 2.9 & & 2.5 & \\
\hline Inter-run \% Bias & & -2.2 & & 1.3 & & 2.7 & & 2.0 & & 0.0 & \\
\hline $\mathrm{n}$ & & 18 & & 18 & & 18 & & 18 & & 18 & \\
\hline
\end{tabular}




\begin{tabular}{llll} 
Table S-2 & \multicolumn{3}{l}{$\begin{array}{l}\text { Post-preparative stability in human plasma sample extracts with the } \\
\text { autosampler set at } \mathbf{5}^{\circ} \mathbf{C}\end{array}$} \\
\hline Storage period / container & $\begin{array}{l}\text { Nominal concentration } \\
(\mathrm{ng} / \mathrm{mL})\end{array}$ & $\begin{array}{l}\text { Measured concentrations } \\
(\mathrm{ng} / \mathrm{mL})\end{array}$ & Mean bias (\%) \\
\hline 5 days & 0.300 & 0.294 & $0.308(4.6)$ \\
& 0.297 & \\
& 0.322 & $37.4(0.6)$ \\
& 0.317 & \\
\hline 37.5 & 37.5 & \\
& 37.2 & 37.2 & \\
& & 37.6 & \\
& & & \\
\end{tabular}

\begin{tabular}{llll} 
Table S-3 & Freeze-thaw stability in human plasma samples stored at or below $\mathbf{- 6 0 ^ { \circ } \mathbf { C }}$ \\
\hline $\begin{array}{llll}\text { Number of freeze-thaw } \\
\text { cycles }\end{array}$ & Nominal concentration $(\mathrm{ng} / \mathrm{mL})$ & Measured concentrations $(\mathrm{ng} / \mathrm{mL})$ & Mean bias (\%) \\
\hline 3 & 0.300 & 0.291 & $0.301(0.3)$ \\
& & 0.322 & \\
\hline 3 & 37.5 & 0.291 & $36.7(-2.1)$ \\
& 36.4 & \\
& 36.7 & 37.1 & \\
\end{tabular}

Table S-4 Stability in spiked human plasma samples stored at room temperature

\begin{tabular}{llll}
\hline Storage period & Nominal concentration $(\mathrm{ng} / \mathrm{mL})$ & Measured concentrations $(\mathrm{ng} / \mathrm{mL})$ & Mean bias $(\%)$ \\
\hline 24 hours & 0.300 & 0.308 & $0.300(0.0)$ \\
& & 0.294 & \\
\cline { 2 - 4 } & \multirow{2}{*}{37.5} & 0.298 & $37.0(-1.3)$ \\
& & 37.4 & \\
\hline
\end{tabular}

Table S-5 Stability in spiked human plasma samples stored at or below $-60^{\circ} \mathrm{C}$

\begin{tabular}{llll}
\hline Storage period & Nominal concentration $(\mathrm{ng} / \mathrm{mL})$ & Measured concentrations $(\mathrm{ng} / \mathrm{mL})$ & Mean bias $(\%)$ \\
\hline \multirow{2}{*}{0.300} & 0.263 & $0.296(-1.3)$ \\
& & 0.282 & \\
& & 0.331 & \\
\cline { 2 - 4 } & & 0.306 & $33.0(-12.0)$ \\
& 37.5 & 32.7 & \\
& 32.4 & \\
& 33.2 & 33.6 & \\
& & & \\
& & & \\
\end{tabular}




\section{Table S-6 Carryover in human plasma blanks following injection of the ULOQ for}

pasireotide

\begin{tabular}{llll}
\hline Pasireotide & Individual peak area at the LLOQ & Blank 1 & Blank 2 \\
\hline Measured peak area & 2378 & 0 & 0 \\
& 2807 & & \\
& 2413 & & \\
2573 & & \\
& 2589 & & \\
& 2204 & 0 & 0 \\
\hline Mean & 2494 & 0 & \\
\hline Carryover (\%) & & & \\
\hline
\end{tabular}

Table S-7 Carryover in human plasma blanks following injection of the ULOQ for $[\mathrm{M}+6]$ pasireotide

\begin{tabular}{llll}
\hline$[\mathrm{M}+6]$ Pasireotide & Individual peak area at the working concentration & Blank 1 & Blank 2 \\
\hline Measured peak area & 48322 & 0 & 0 \\
& 51409 & & \\
& 50291 & & \\
& 50098 & & \\
& 49927 & & \\
& 51475 & & \\
\hline Mean & 50254 & 0 & 0 \\
\hline Carryover $(\%)$ & & & \\
\hline
\end{tabular}

Table S-8 Stability of pasireotide in stock solution

\begin{tabular}{lllll}
\hline Injection \# & LC-MS response & & & \\
\cline { 2 - 5 } & Solution 1 & Solution 2 & Solution 3 & Solution 4 \\
\hline 1 & 12.61 & 13.11 & 13.22 & 13.18 \\
2 & 12.51 & 13.03 & 13.25 & 13.16 \\
3 & 12.54 & 13.02 & 13.10 & 13.51 \\
4 & 12.32 & 13.05 & 13.02 & 13.01 \\
5 & 12.43 & 13.09 & 13.12 & 13.15 \\
6 & 12.45 & 13.01 & 13.04 & 13.20 \\
\hline Mean response & $\mathbf{1 2 . 4 7 9}$ & $\mathbf{1 3 . 0 5 2}$ & $\mathbf{1 3 . 1 2 4}$ & $\mathbf{1 3 . 2 0 4}$ \\
\hline & \% Difference & Duration (Day) & \\
\hline Solution 1 vs. Solution 2 & -4.4 & 0 Days & \\
Solution 1 vs. Solution 3 & -4.9 & 268 Days & \\
Solution 1 vs. Solution 4 & -5.5 & 268 Days & \\
Solution 2 vs. Solution 3 & -0.5 & 268 Days & \\
Solution 2 vs. Solution 4 & -1.1 & 268 Days & \\
Solution 3 vs. Solution 4 & -0.6 & 0 Days & \\
\hline
\end{tabular}


Table S-9 Matrix effect for pasireotide

\begin{tabular}{|c|c|c|c|c|c|c|c|c|c|}
\hline & \multicolumn{3}{|c|}{37.5 ng/mL (QC High) } & \multicolumn{3}{|c|}{15.0 ng/mL (QC Mid) } & \multicolumn{3}{|c|}{0.300 ng/mL (QC Low) } \\
\hline & $\begin{array}{l}\text { Expected } \\
\text { area }^{1}\end{array}$ & $\begin{array}{l}\text { Measured } \\
\text { area }^{2}\end{array}$ & $\mathrm{MF}^{*}$ & $\begin{array}{l}\text { Expected } \\
\text { area }^{1}\end{array}$ & $\begin{array}{l}\text { Measured } \\
\text { area }^{2}\end{array}$ & $\mathrm{MF}^{*}$ & $\begin{array}{l}\text { Expected } \\
\text { area }^{1}\end{array}$ & $\begin{array}{l}\text { Measured } \\
\text { area }^{2}\end{array}$ & $\mathrm{MF}^{*}$ \\
\hline \multirow[t]{3}{*}{ BRH898301 } & 875603 & 792942 & 0.906 & 357402 & 357826 & 1.00 & 8131 & 6972 & 0.857 \\
\hline & 928597 & 856075 & 0.922 & 362542 & 334865 & 0.924 & 7180 & 6883 & 0.959 \\
\hline & 897021 & 824779 & 0.919 & 395747 & 337561 & 0.853 & 7830 & 6301 & 0.805 \\
\hline Mean MF & & & 0.916 & & & 0.926 & & & 0.874 \\
\hline CV\% & & & 1.0 & & & 8.0 & & & 9.0 \\
\hline \multirow[t]{3}{*}{ BRH883823 } & 875603 & 856584 & 0.978 & 357402 & 360520 & 1.01 & 8131 & 6780 & 0.834 \\
\hline & 928597 & 936831 & 1.01 & 362542 & 431708 & 1.19 & 7180 & 7476 & 1.04 \\
\hline & 897021 & 939814 & 1.05 & 395747 & 350826 & 0.886 & 7830 & 6525 & 0.833 \\
\hline Mean MF & & & 1.01 & & & 1.03 & & & 0.903 \\
\hline CV\% & & & 3.4 & & & 14.9 & & & 13.3 \\
\hline \multirow[t]{3}{*}{ BRH880427 } & 875603 & 894823 & 1.02 & 357402 & 367277 & 1.03 & 8131 & 7565 & 0.930 \\
\hline & 928597 & 879255 & 0.947 & 362542 & 344072 & 0.949 & 7180 & 7080 & 0.986 \\
\hline & 897021 & 828419 & 0.924 & 395747 & 345608 & 0.873 & 7830 & 7004 & 0.895 \\
\hline Mean MF & & & 0.964 & & & 0.950 & & & 0.937 \\
\hline CV\% & & & 5.3 & & & 8.1 & & & 4.9 \\
\hline \multirow[t]{3}{*}{ BRH794873 } & 875603 & 838927 & 0.958 & 357402 & 327918 & 0.918 & 8131 & 7092 & 0.872 \\
\hline & 928597 & 850670 & 0.916 & 362542 & 346922 & 0.957 & 7180 & 7310 & 1.02 \\
\hline & 897021 & 838330 & 0.935 & 395747 & 364293 & 0.921 & 7830 & 7159 & 0.914 \\
\hline Mean MF & & & 0.936 & & & 0.932 & & & 0.935 \\
\hline CV\% & & & 2.3 & & & 2.4 & & & 8.0 \\
\hline \multirow[t]{3}{*}{ BRH800130 } & 875603 & 918312 & 1.05 & 357402 & 398145 & 1.11 & 8131 & 7724 & 0.950 \\
\hline & 928597 & 873182 & 0.940 & 362542 & 343170 & 0.947 & 7180 & 6843 & 0.953 \\
\hline & 897021 & 898411 & 1.002 & 395747 & 338253 & 0.855 & 7830 & 7016 & 0.896 \\
\hline Mean MF & & & 0.997 & & & 0.972 & & & 0.933 \\
\hline CV\% & & & 5.5 & & & 13.5 & & & 3.4 \\
\hline \multirow[t]{3}{*}{ BRH800131 } & 875603 & 851829 & 0.973 & 357402 & 254289 & 0.711 & 8131 & 7142 & 0.878 \\
\hline & 928597 & 841694 & 0.906 & 362542 & 328938 & 0.907 & 7180 & 7117 & 0.991 \\
\hline & 897021 & 867351 & 0.967 & 395747 & 337251 & 0.852 & 7830 & 6546 & 0.836 \\
\hline Mean MF & & & 0.949 & & & 0.824 & & & 0.902 \\
\hline CV\% & & & 3.9 & & & 12.3 & & & 8.9 \\
\hline
\end{tabular}




\begin{tabular}{|c|c|c|c|c|c|c|c|c|c|}
\hline & \multicolumn{3}{|c|}{$37.5 \mathrm{ng} / \mathrm{mL}$ (QC High) } & \multicolumn{3}{|c|}{$15.0 \mathrm{ng} / \mathrm{mL}$ (QC Mid) } & \multicolumn{3}{|c|}{0.300 ng/mL (QC Low) } \\
\hline & $\begin{array}{l}\text { Expected } \\
\text { area }^{1}\end{array}$ & $\begin{array}{l}\text { Measured } \\
\text { area }^{2}\end{array}$ & $\mathrm{MF}^{*}$ & $\begin{array}{l}\text { Expected } \\
\text { area }^{1}\end{array}$ & $\begin{array}{l}\text { Measured } \\
\text { area }^{2}\end{array}$ & $\mathrm{MF}^{*}$ & $\begin{array}{l}\text { Expected } \\
\text { area }^{1}\end{array}$ & $\begin{array}{l}\text { Measured } \\
\text { area }^{2}\end{array}$ & $\mathrm{MF}^{\star}$ \\
\hline Overall mean MF at each level & & & 0.962 & & & 0.939 & & & 0.914 \\
\hline CV\% & & & 3.8 & & & 7.2 & & & 2.8 \\
\hline Overall mean MF across all levels & & & 0.938 & & & & & & \\
\hline CV\% & & & 5.1 & & & & & & \\
\hline
\end{tabular}

${ }^{1}$ Area of the analyte in solution

${ }^{2}$ Area of the analyte spiked into an extracted blank sample

*Matrix Factor

\section{Table S-10 Recovery for pasireotide}

\begin{tabular}{|c|c|c|c|c|c|c|c|c|c|}
\hline & \multicolumn{9}{|c|}{ PASIREOTIDE nominal concentration (ng/mL) } \\
\hline & \multicolumn{3}{|c|}{0.300 ng/mL (QCs low) } & \multicolumn{3}{|c|}{$15.0 \mathrm{ng} / \mathrm{mL}$ (QCs mid) } & \multicolumn{3}{|c|}{$37.5 \mathrm{ng} / \mathrm{mL}$ (QCs high) } \\
\hline & \multicolumn{9}{|c|}{ PASIREOTIDE areas and recoveries } \\
\hline & $\begin{array}{l}\text { Expected } \\
\text { area }^{1}\end{array}$ & $\begin{array}{l}\text { Measured } \\
\text { area }^{2}\end{array}$ & $\begin{array}{l}\text { Recovery } \\
(\%)\end{array}$ & $\begin{array}{l}\text { Expected } \\
\text { area }^{1}\end{array}$ & $\begin{array}{l}\text { Measured } \\
\text { area }^{2}\end{array}$ & $\begin{array}{l}\text { Recovery } \\
(\%)\end{array}$ & $\begin{array}{l}\text { Expected } \\
\text { area }^{1}\end{array}$ & $\begin{array}{l}\text { Measured } \\
\text { area }^{2}\end{array}$ & $\begin{array}{l}\text { Recovery } \\
(\%)\end{array}$ \\
\hline \multirow[t]{3}{*}{$\mathrm{BRH} 1065428$} & 6119 & 3051 & 49.9 & 320931 & 154022 & 48.0 & 870616 & 382260 & 43.9 \\
\hline & 6212 & 2811 & 45.3 & 307838 & 146521 & 47.6 & 770439 & 397179 & 51.6 \\
\hline & 5464 & 2977 & 54.5 & 317777 & 160318 & 50.4 & 800712 & 400001 & 50.0 \\
\hline Mean & & & 49.9 & & & 48.7 & & & 48.5 \\
\hline CV\% & & & 9.2 & & & 3.2 & & & 8.3 \\
\hline Overall mean & & & & & & 49.0 & & & \\
\hline CV\% & & & & & & 1.5 & & & \\
\hline
\end{tabular}

${ }^{1}$ Area of the analyte spiked into an extracted blank

${ }^{2}$ Area of the analyte spiked into the blank matrix and extracted 
Figure S-1 A comparison of LC-DMS-MIM response for pasireotide with and without chemical modifier isopropanol

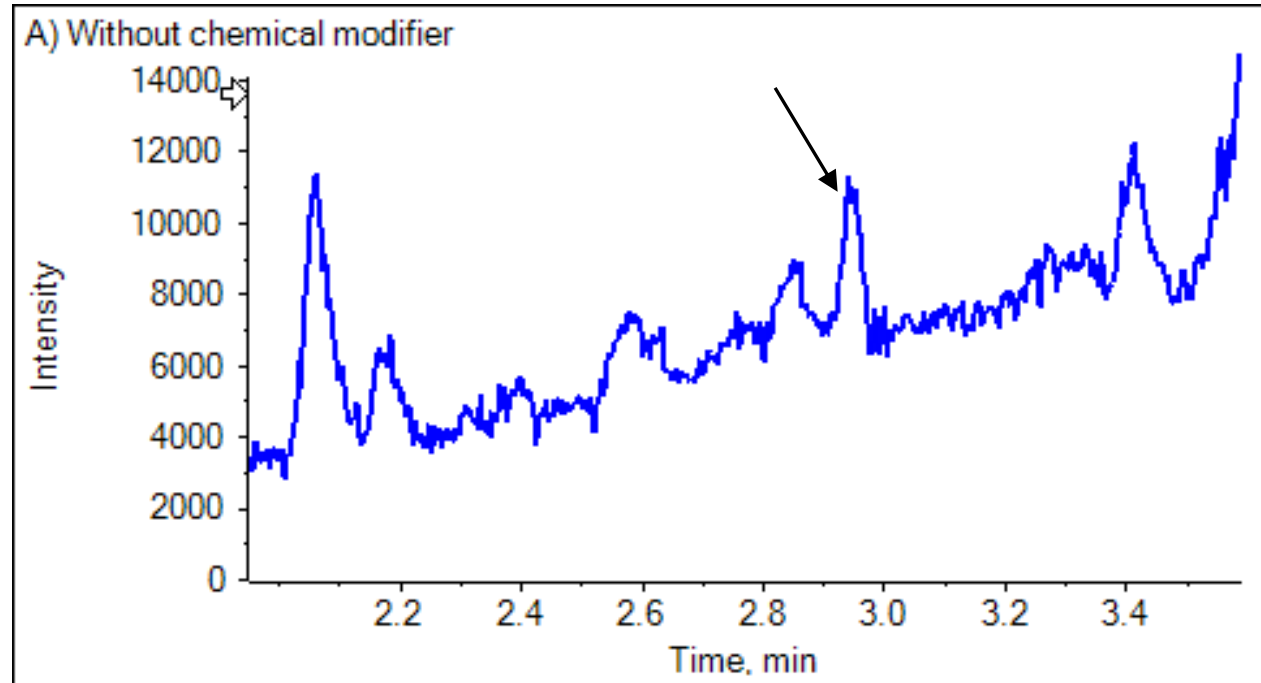

B) IPA as chemical modifier

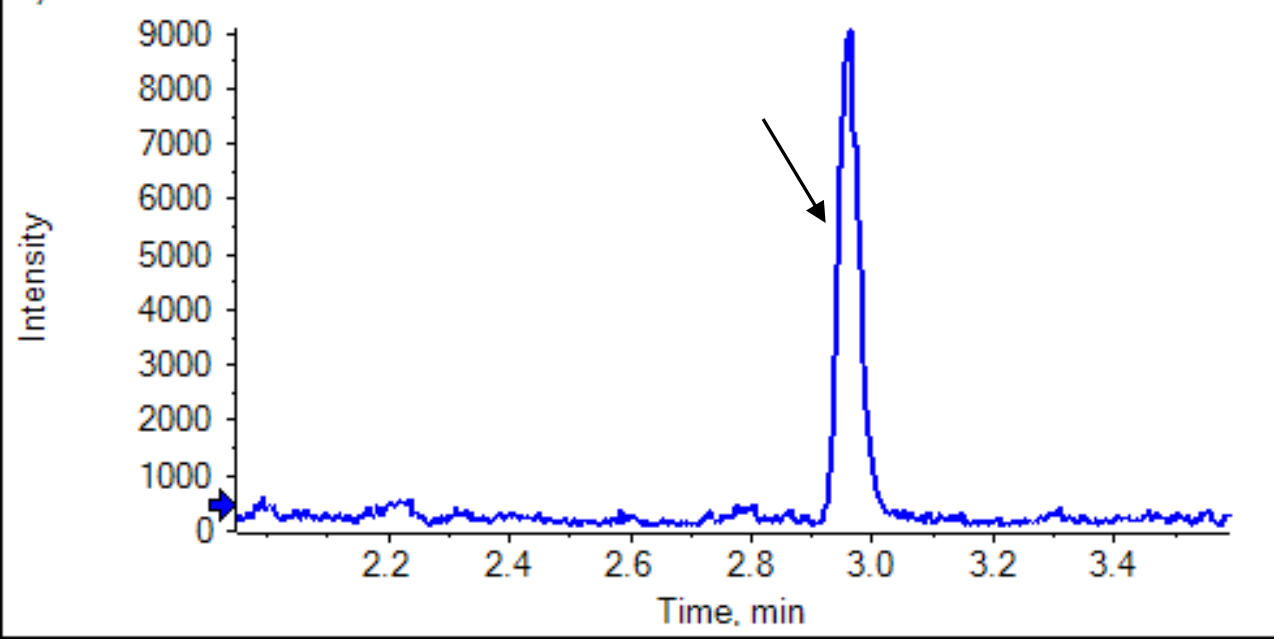

